\title{
Modulation of Substrate Specificity within the Amino Acid Editing Site of Leucyl-tRNA Synthetase ${ }^{\dagger}$
}

\author{
Yuxin Zhai, ${ }^{\ddagger}$ Mir Hussain Nawaz,$^{\S}$ Keun Woo Lee," Erin Kirkbride, James M. Briggs, and Susan A. Martinis*,§ \\ Department of Biology and Biochemistry, University of Houston, Houston, Texas 77204-5001 \\ Received August 28, 2006; Revised Manuscript Received January 13, 2007
}

\begin{abstract}
The aminoacyl-tRNA synthetases covalently link transfer RNAs to their cognate amino acids. Some of the tRNA synthetases have evolved editing mechanisms to ensure fidelity in this first step of protein synthesis. The amino acid editing site for leucyl- (LeuRS) and isoleucyl- (IleRS) tRNA synthetases reside within homologous CP1 domains. In each case, a threonine-rich peptide and a second conserved GTG region that are separated by about 100 amino acids comprise parts of the hydrolytic editing site. While a number of sites are conserved between these two enzymes and likely confer a commonality to the mechanisms, some positions are idiosyncratic to LeuRS or IleRS. Herein, we provide evidence that a conserved arginine and threonine at respective sites in LeuRS and IleRS diverged to confer amino acid substrate recognition. This site complements other sites in the amino acid binding pocket of the editing active site of Escherichia coli LeuRS, including $\mathrm{Thr}^{252}$ and $\mathrm{Val}^{338}$, which collectively fine-tune amino acid specificity to confer fidelity.
\end{abstract}

The fidelity of protein synthesis is dependent on the aminoacyl-tRNA synthetases $\left(\mathrm{aaRSs}^{1}\right)$, which link amino acids to their cognate transfer RNAs (tRNAs) $(1,2)$. There are typically up to twenty types of aaRSs per cell or organelle. Each corresponds to one of the standard amino acids that are incorporated into proteins during ribosomebased translation. The aaRSs catalyze the formation of aminoacyl-tRNA in a two-step reaction mechanism:

$$
\begin{aligned}
& \text { amino acid }(\mathrm{AA})+ \text { ATP } \rightleftarrows \\
& \text { AA-AMP }+ \text { pyrophosphate }\left(\mathrm{PP}_{\mathrm{i}}\right) \\
& \text { AA-AMP }+ \text { tRNA } \rightarrow \text { AA-tRNA }+ \text { AMP }
\end{aligned}
$$

The charged tRNA is then bound by an elongation factor and transported to the ribosome where the amino acid is incorporated into the nascent peptide chain.

Because of structural similarities between some amino acids, a number of aaRSs have difficulty completely dis-

$\doteqdot$ This work was supported by The Robert A. Welch Foundation (E1404) and the National Institutes of Health (GM63789). We also acknowledge the Texas Learning and Computation Center and the Institute for Molecular Design at the University of Houston for computer time and software.

* To whom correspondence should be addressed: Department of Biochemistry, University of Illinois, 419 RAL, Box B-4, 600 South Mathews Ave., Urbana, IL 61801-3732. Phone: 217-244-2405. Fax: 217-244-5858. E-mail: martinis@life.uiuc.edu.

$\doteqdot$ Present address: Department of Biochemistry and Molecular Biology, University of Texas Medical School at Houston, 6431 Fannin, Houston, TX 77030.

$\S$ Present address: Department of Biochemistry, University of Illinois, 419 RAL, Box B-4, 600 South Mathews Ave., Urbana, IL 61801-3732.

"Present address: Division of Applied Life Science, Environmental Biotechnology National Core Research Center, Gyeongsang National University, 900 Gazwa-Dong, Jinju 660-701, Republic of Korea.

${ }^{1}$ Abbreviations: aaRS, aminoacyl-tRNA synthetase; CP1, connective peptide 1; DTT, D,L-dithiothreitol; Nva, norvaline; LeuRS, leucyl-tRNA synthetase; IleRS, isoleucyl-tRNA synthetase; X\#\#\#, X residue at position \#\# is substituted by $\mathrm{Y}$ amino acid. criminating their cognate substrate from other similar amino acids during catalysis (3). To minimize the production of mischarged tRNAs, many aaRSs have developed amino acid editing activities. A "double-sieve" mechanism (4-6) introduces two separate amino acid binding sieves or pockets with different strategies for recognition to enhance fidelity. In the synthetases, the "coarse sieve" corresponds to the amino acid activation and aminoacylation active site. It binds cognate amino acid, as well as related analogues that can also fit in the amino acid binding pocket to form activated aminoacyl-adenylates and charged tRNAs. A second "fine sieve" can be correlated to the amino acid editing site. Its primary purpose is to clear mistakes created in the aminoacylation active site, but also must prevent the correct amino acid from being hydrolyzed from the aminoacyl-adenylate intermediate or charged tRNA.

The aminoacylation active sites of the class I editing leucyl- (LeuRS) (7), isoleucyl- (IleRS) (8), and valyl-tRNA synthetases (ValRS) (9) are located within the canonical class I Rossmann fold core of the synthetase. The editing sieves for LeuRS, IleRS, and ValRS reside in separate homologous domains called CP1 (10-12). The editing site is marked by a universally conserved aspartic acid that anchors the common amino moiety of the bound amino acid via a hydrogen bond $(13,14)$. ValRSs and bacterial LeuRSs also have a highly conserved second aspartic acid that is three amino acids upstream and in close tertiary proximity to the universally conserved aspartic acid. In cocrystal structures of Thermus thermophilus LeuRS, this Asp ${ }^{344}$ interacts via a bridging water molecule in a network of hydrogen bonds to the universally conserved aspartic acid, the bound substrate's amino moiety, and also the oxygen atom of the ester linkage (14). In the "pre-transfer editing complex" of LeuRS (14) and a model for ValRS (15), this aspartic acid forms a hydrogen bond with the $3^{\prime}$ hydroxyl of the bound ribose. 
Each CP1 domain also shares a threonine-rich region that marks a portion of the editing site where catalysis occurs. Mutation of a conserved $\mathrm{Thr}^{252}$ residue to serine or alanine within the threonine-rich region of Escherichia coli LeuRS uncouples amino acid specificity such that correctly charged leucine is rapidly hydrolyzed from $\operatorname{tRNA}^{\mathrm{Leu}}(12,16)$. Insertion of bulkier residues including tyrosine or phenylalanine blocks amino acid editing $(17,18)$. The X-ray crystal structure of $T$. thermophilus LeuRS bound to either the preor post-transfer editing substrate analogue supports that the threonine side chain sterically blocks the $\gamma$-methyl group of the leucine side chain (14) and therefore prevents hydrolytic editing of the correctly charged Leu-tRNA ${ }^{\text {Leu }}$.

The X-ray crystal structures (14) also suggested that a valine $\left(\mathrm{Val}^{340}\right.$ in $T$. thermophilus LeuRS) within a second conserved CP1-based peptide might contribute to leucine specificity (Figure 1). In addition, conserved sites within these two LeuRS peptides that comprise the amino acid binding pocket of the editing site (including $\mathrm{Thr}^{247}$, $\mathrm{Thr}^{248}$, $\mathrm{Thr}^{252}, \mathrm{Ile}^{337}, \mathrm{Pro}^{341}, \mathrm{Ala}^{342}$, and $\mathrm{His}^{343}$ ) are also highly conserved in IleRS. However, some neighboring sites are also conserved in LeuRS and IleRS, but have different amino acids at the corresponding sites in these respective enzymes (Figure 1D). We hypothesized that these positions might be important to amino acid recognition in the editing site that contributes significantly to the fidelity of protein synthesis. We mutationally analyzed these two peptides that line the amino acid binding pocket of LeuRS to characterize those that play a role in editing specificity.

\section{EXPERIMENTAL PROCEDURES}

Materials. Restriction enzymes $D p n I$ and Bst NI were acquired from Promega (Madison, WI) and New England BioLabs Inc. (Beverly, MA) respectively. Cloned Pfu DNA polymerase and dNTP mix were purchased from Stratagene (La Jolla, CA). Tritium-labeled amino acids were obtained from Amersham Pharmacia Biotech (Piscataway, NJ). Crude E. coli $\mathrm{tRNA}^{\mathrm{Leu}}$ was from Hoffmann-La Roche Ltd (Basel, Switzerland). For in vitro transcription, a total of $450 \mu \mathrm{g}$ of plasmid ptDNAleu (19) containing the gene for E. coli tRNA ${ }^{\text {Leu }}$ was digested overnight with $25 \mathrm{U} B s t \mathrm{~N} 1$ in a $1 \mathrm{~mL}$ reaction at $60{ }^{\circ} \mathrm{C}$ and then used as template (20). E. coli $\mathrm{tRNA}_{\mathrm{UAA}}^{\mathrm{Leu}}\left(\mathrm{tRNA}^{\mathrm{Leu}}\right)$ was in vitro transcribed, purified, and aminoacylated using a published procedure (21). Oligonucleotide primer synthesis was performed by MWG Biotech (High Point, NC).

Mutagenesis and Purification of E. coli LeuRS. Wild type LeuRS was mutated to R249F, R249A, R249F/T252A, M336F, M336A, M336F/T252A, V338A, V338F, V338F/ T252A, V338D, V338E, V338L, R249T, D251W, and R249T/D251W using polymerase chain reaction (PCR)-based reaction mutagenesis and the plasmid p15ec3-1 (22) as template as described previously (21). The mutant plasmids were selected in E. coli DH5 $\alpha$ strain. The mutation in the isolated plasmid was confirmed by DNA sequencing at Lone Star Labs (Houston, TX).

Protein expression for mutant and wild type plasmids was carried out in E. coli BL21 strain (Stratagene, La Jolla, CA) as described previously (21). Each protein contained an N-terminal fused six-histidine tag and was purified to homogeneity via affinity chromatography using HIS-Select
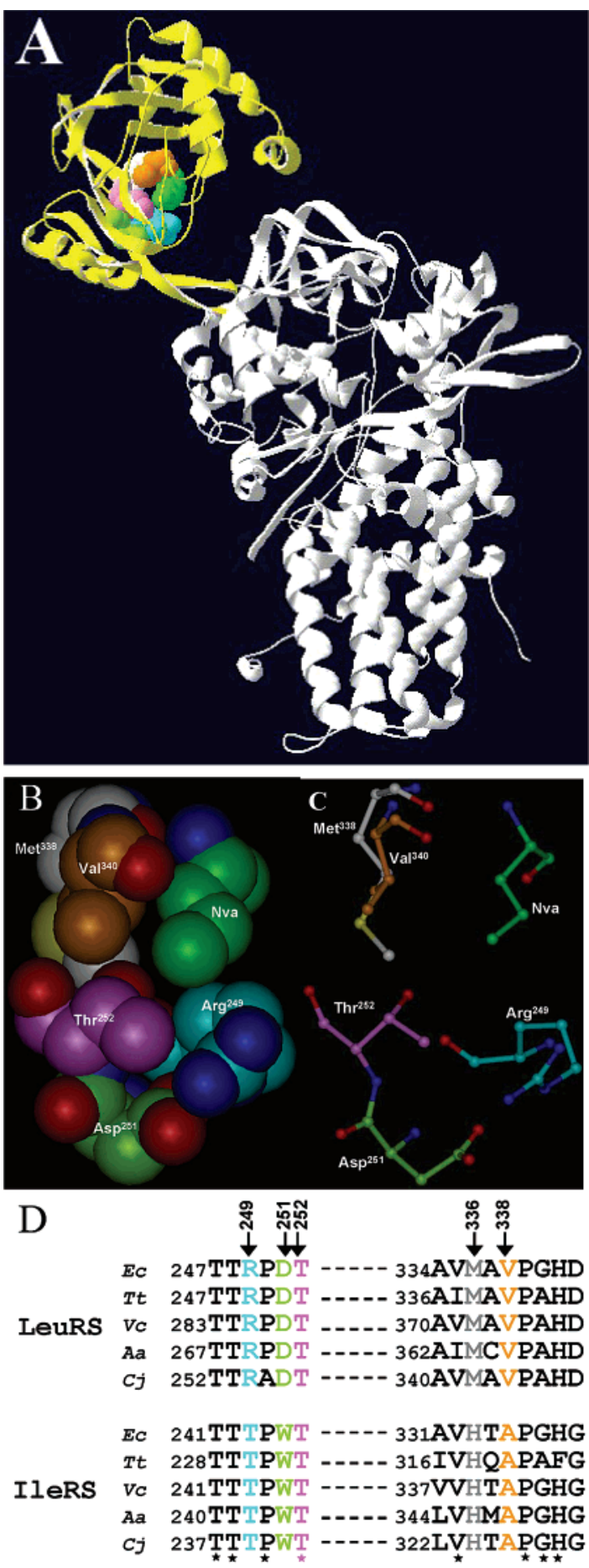

FIGURE 1: Tertiary and primary structure analysis of the amino acid editing site. (A) X-ray crystal structure of T. thermophilus LeuRS (14). The main body of the enzyme is colored in white, and the CP1 domain is yellow. The amino acid binding pocket of T. thermophilus LeuRS with norvaline (dark green) of the Nva2AA post-transfer editing analogue bound (14) is highlighted as a spacefilling (B) and ball and stick model (C). The atoms are colored as follows: oxygen, red; sulfur, yellow; and nitrogen, blue; $\operatorname{Arg}^{249}$ carbons, light blue; Asp ${ }^{251}$ carbons, light green; Thr ${ }^{252}$ carbons, pink; $\mathrm{Met}^{338}$ (equivalent to $\mathrm{Met}^{336}$ in E. coli LeuRS) carbons, gray; and $\mathrm{Val}^{340}$ (equivalent to $\mathrm{Val}^{338}$ in $E$. coli LeuRS) carbons, orange. (D) Primary sequence alignment of LeuRS and IleRS amino acid editing site. The $\mathrm{Arg}^{249}, \mathrm{Asp}^{251}, \mathrm{Thr}^{252}$, $\mathrm{Met}^{336}$, and $\mathrm{Val}^{338}$ residues are colored in light blue, green, pink, gray, and orange respectively. Asterisks indicate residues that are highly conserved or homologous across both the LeuRS and IleRS enzymes. Abbreviations are as follows: Ec, Escherichia coli; Tt, Thermus thermophilus; Vc, Vibrio cholerae; Aa, Aquifex aeolicus; and $\mathrm{Cj}$, Campylobacter jejuni. 
HF Nickel Affinity Gel (Sigma, St. Louis, MO) (21). The final stock concentrations of proteins that were used in enzymatic assays were determined by a Bio-Rad Protein Assay as described in the commercial protocol.

Enzyme Assays. Aminoacylation reactions contained 60 $\mathrm{mM}$ Tris, $\mathrm{pH} 7.5,10 \mathrm{mM} \mathrm{MgCl} 2,1 \mathrm{mM}$ dithiothreitol (DTT), and $4 \mathrm{mg} / \mathrm{mL}$ crude $E$. coli tRNA $^{\text {Leu }}$. The enzyme and amino acid concentrations are indicated in the appropriate figure legend. The reactions were initiated by adding $4 \mathrm{mM}$ ATP. Aliquots for each reaction were quenched at specific time points and processed as previously described (21).

Post-transfer editing assays were carried out using in vitro transcribed $E$. coli RRNA $^{\text {Leu }}$ that was aminoacylated with $\left[{ }^{3} \mathrm{H}\right]$-isoleucine or $\left[{ }^{3} \mathrm{H}\right]$-leucine as described previously $(21)$. Each reaction contained $60 \mathrm{mM}$ Tris, $\mathrm{pH} 7.5,10 \mathrm{mM} \mathrm{MgCl}_{2}$, $1 \mathrm{mM}$ DTT, and $0.8 \mu \mathrm{M}$ Leu- or Ile-tRNA ${ }^{\text {Leu }}$. Concentrations of Leu-tRNA ${ }^{\text {Leu }}$ and Ile-tRNA ${ }^{\text {Leu }}$ were varied from 0.1 to $1.0 \mu \mathrm{M}$ to determine kinetic parameters. Enzyme concentrations were optimized for kinetic analysis of Ile-tRNA ${ }^{\mathrm{Leu}}$ hydrolysis as follows: WT, $5 \mathrm{nM}$; V338D, $10 \mathrm{nM}$; V338E, $15 \mathrm{nM}$; V338F, $1 \mu \mathrm{M}$; V338A, $10 \mathrm{nM}$; and V338L, $15 \mathrm{nM}$. In the case of Leu-tRNA ${ }^{\text {Leu }}$ editing kinetic measurements, the enzyme concentrations were $25 \mathrm{nM}$ wild type and 15 nM V338A mutant LeuRS: The apparent values for $K_{\mathrm{m}}$ and $k_{\text {cat }}$ and their standard deviations were determined using the Enzyme Kinetics Module 1.1 of SigmaPlot software.

\section{RESULTS}

Mutational Analysis of the Editing Site's Amino Acid Binding Pocket. The X-ray crystal structures have been solved for LeuRS complexed to pre- and post-transfer editing analogues (14) as well as the tRNA bound in the editing complex $(23,24)$. A universally conserved aspartic acid (Asp $\mathrm{s}^{345}$ in E. coli LeuRS) as well as a highly conserved aspartic acid ( $\mathrm{Asp}^{342}$ in E. coli LeuRS) that is three amino acids upstream interact and anchor the backbone of the amino acid and the ribose hydroxyl groups (14). In addition, two conserved threonine residues ( $\mathrm{Thr}^{247}$ and $\mathrm{Thr}^{248}$ in E. coli LeuRS) also interact with the ribose hydroxyl groups and the covalent link to the amino acid (21). The side chains of $\mathrm{Arg}^{249}, \mathrm{Thr}^{252}$, Met ${ }^{336}$, and $\mathrm{Val}^{338}$ line the amino acid binding pocket of the editing site and were hypothesized to confer specificity (14) (Figure 1). Previously, we determined that the $\mathrm{Thr}^{252}$ residue in E. coli LeuRS is a critical discriminator that blocks leucine from the editing site (12). Introduction of an alanine or serine uncouples specificity and allows hydrolysis of the Leu-tRNA ${ }^{\text {Leu }}(12,16)$. Bulky mutations have also been substituted at the $\mathrm{Thr}^{252}$ site to fill in the amino acid binding pocket and block editing activity (17, 18).

We combined the T252A mutation that uncouples specificity and scanned for effects on editing with bulky phenylalanine substitutions at $\operatorname{Arg}^{249}, \mathrm{Met}^{336}$, and $\mathrm{Val}^{338}$. We hypothesized that introduction of phenylalanine at sites where the side chains actually lined the amino acid binding pocket would block substrate binding and impede overall editing activity to rescue the T252A phenotype. As shown in Figure 2 , introduction of the large aromatic residue at $\operatorname{Arg}^{249}$ or $\mathrm{Val}^{338}$ rescued leucylation activity of the T252A mutation, suggesting that editing activity of Leu-tRNA ${ }^{\text {Leu }}$ is decreased or abolished by $\mathrm{R} 249 \mathrm{~F}$ and $\mathrm{V} 338 \mathrm{~F}$ such that the robust
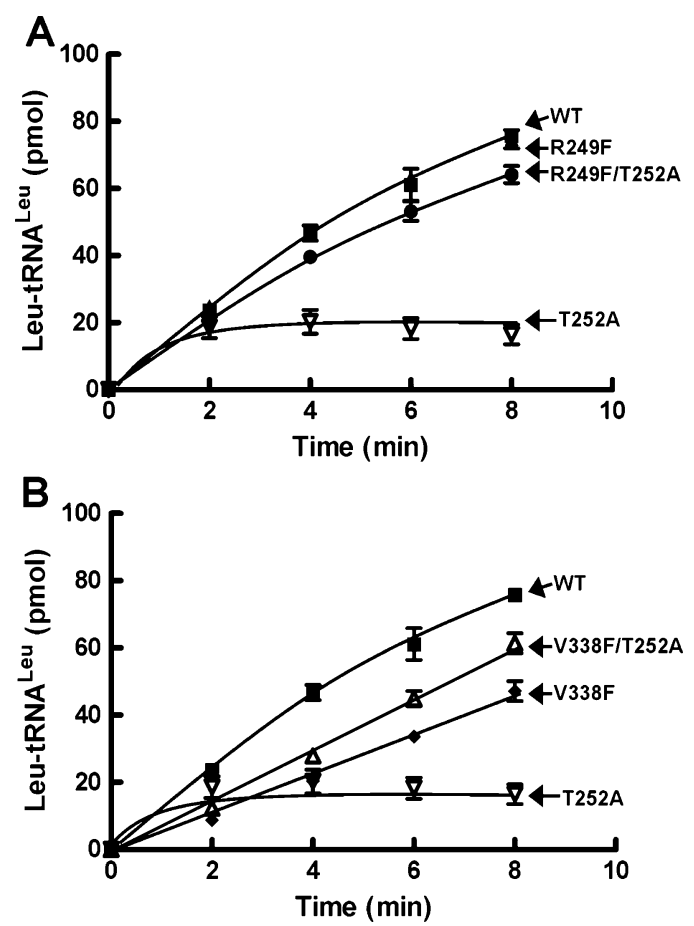

FIGURE 2: Leucylation of tRNA ${ }^{\text {Leu }}$ by wild type and mutant enzymes. The $\mathrm{Arg}^{249}(\mathrm{~A})$ and $\mathrm{Val}^{338}$ (B) single and double mutations with T252A are compared to the wild type enzyme and T252A LeuRS mutant. Each aminoacylation reaction was carried out in the presence of $4 \mathrm{mg} / \mathrm{mL}$ crude $E$. coli tRNA, $25.5 \mu \mathrm{M}$ leucine $(150 \mu \mathrm{Ci} / \mathrm{mL})$, and $500 \mathrm{nM}$ enzyme. Symbols represent the following wild type and mutant LeuRSs: wild type (WT), $\mathbf{\square}$;

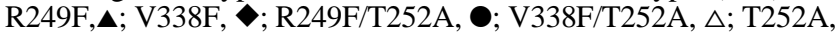
$\nabla$. Error bars are the result of three reactions and indicated for each time point.

leucylation activity could now be detected. Substitution of $\operatorname{Arg}^{249}$ with an alanine shows little difference in leucine (12) or isoleucine specificity (data not shown). Similar to bulky mutations at the $\mathrm{Thr}^{252}$ position $(17,18)$, we propose that the second phenylalanine mutation at $\mathrm{Arg}^{249}$ or $\mathrm{Val}^{338}$ fills in the amino acid binding pocket and prevents leucine and other amino acids from binding to the more open T252A amino acid binding pocket within the editing site and bars editing of Leu-tRNA ${ }^{\text {Leu }}$.

The M336F/T252A double LeuRS mutant exhibited only slightly increased leucylation activity relative to the T252A single mutation (data not shown). A single M336A mutant enzyme did show a small increase in leucine editing activity, suggesting that it can enlarge the amino acid binding pocket to accommodate the correctly charged leucine. These combined results suggest that the conserved Met ${ }^{336}$ plays a relatively subtle role in amino acid discrimination compared to $\mathrm{Thr}^{252}$, $\mathrm{Arg}^{249}$, and $\mathrm{Val}^{338}$.

Val $^{338}$ Is Secondary to Thr ${ }^{252}$ as a Fine Discriminator of Leucine. $\mathrm{Val}^{338}$ has been proposed to aid $\mathrm{Thr}^{252}$ in blocking leucine from the editing site by directly clashing with one of the $\gamma$-methyl groups of leucine (14). Computational modeling also suggested that $\mathrm{Val}^{338}$ might play a role in maintaining the geometry of the amino acid editing site to confer specificity and activity (16). We further analyzed $\mathrm{Val}^{338}$ by introducing a series of single mutations that included V338D, V338E, V338F, V338A, and V338L and tested each of these mutants for aminoacylation activity. Only the V338A mutant LeuRS appeared to affect leucylation 


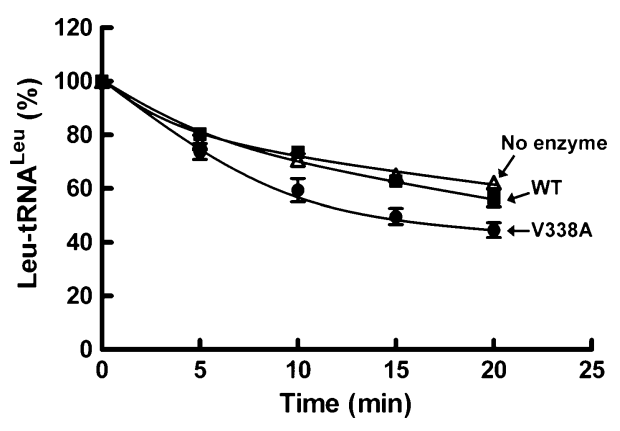

FIGURE 3: Wild type and V338 mutant LeuRS editing activity of

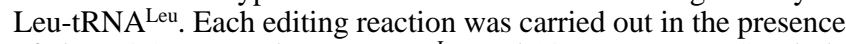
of about $0.2 \mu \mathrm{M}$ crude Leu-tRNA ${ }^{\text {Leu }}$ and $10 \mathrm{nM}$ enzyme. Symbols are represented as follows: WT, $\mathbf{\square}$; V338A, $\mathbf{\bullet}$; and no enzyme, $\triangle$. Error bars are the result of three reactions and indicated for each time point.

Table 1: Apparent Kinetic Constants of Wild Type and Mutant Enzymes for Post-Transfer Editing Activity of Leu-tRNA ${ }^{\text {Leu } a}$

\begin{tabular}{lll}
\hline \multicolumn{1}{c}{ constants } & \multicolumn{1}{c}{ WT } & \multicolumn{1}{c}{ V338A } \\
\hline$K_{\mathrm{M}}(\mu \mathrm{M})$ & $0.7 \pm 0.4$ & $0.9 \pm 0.3$ \\
$k_{\text {cat }}\left(\mathrm{s}^{-1}\right)$ & $0.12 \pm 0.04$ & $0.4 \pm 0.07$ \\
$k_{\text {cat }} / K_{\mathrm{M}}\left(\mu \mathrm{M}^{-1} \mathrm{~s}^{-1}\right)$ & 0.2 & 0.4 \\
\hline
\end{tabular}

${ }^{a}$ These kinetic measurements are apparent values.

activity of crude tRNA ${ }^{\text {Leu }}$ (see Supporting Information), although it did not decrease yields of Leu-tRNA ${ }^{\text {Leu }}$ to the level generated by the T252A mutant LeuRS. We measured the editing activity of Leu-tRNA ${ }^{\text {Leu }}$ of the V338A LeuRS mutant and determined that there was only a very small 2 -fold increase of hydrolysis of the correctly charged LeutRNA $^{\text {Leu }}$, compared to wild type LeuRS (Figure 3). Previously, we have shown that a much greater threshold of editing must occur to correct mischarging activity (17). The apparent $k_{\text {cat }} / K_{\mathrm{M}}$ of the wild type and V338A LeuRSs using Leu-tRNA ${ }^{\text {Leu }}$ as a substrate were $0.2 \mu \mathrm{M}^{-1} \mathrm{~s}^{-1}$ and $0.4 \mu \mathrm{M}^{-1}$ $\mathrm{s}^{-1}$ respectively (Table 1 ). Compared to a 24 -fold increase in hydrolytic activity for the T252A mutant LeuRS (16), we propose that the significance of the $\mathrm{Val}^{338}$ site's role in blocking leucine from the editing active site is much less, if at all, significant.

Each of the $\mathrm{Val}^{338}$ single mutant LeuRSs was also tested for mischarging activity. As found for the V338F/T252A double mutant, the single introduction of the bulky phenylalanine residue nearly abolished post-transfer editing activity and facilitated mischarging of both isoleucine and valine to tRNA $^{\text {Leu }}$ (Figure 4). The apparent $k_{\text {cat }} / K_{\mathrm{M}}$ of the V338F mutant LeuRS was reduced more than 2000-fold to 0.001 $\mu \mathrm{M}^{-1} \mathrm{~s}^{-1}$ (Table 2), while the apparent $k_{\text {cat }} / K_{\mathrm{M}}$ of the $\mathrm{T} 252 \mathrm{~F}$ LeuRS mutant was reduced only 20 -fold to $0.1 \mu \mathrm{M}^{-1} \mathrm{~s}^{-1}$ (16). Our combined results with the V338A and T252A mutant enzymes showed a 2- (Table 1) and 24-fold (16) decrease respectively in the apparent $k_{\text {cat }} / K_{\mathrm{M}}$ of Leu-tRNA ${ }^{\text {Leu }}$ hydrolysis in the editing site. Thus, we hypothesize that the primary role of $\mathrm{Thr}^{252}$ is to block cognate leucine from binding in the editing site to prevent hydrolysis (12), while $\mathrm{Val}^{338}$ is more important in accommodating the side chains of the targeted amino acids, in general, for editing. Similar to the $\mathrm{Thr}^{252}$ site (16), other mutations at $\mathrm{Val}^{338}$ can serve as adequate substitutes. However, the high conservation at both of these positions strongly suggests that threonine at the 252
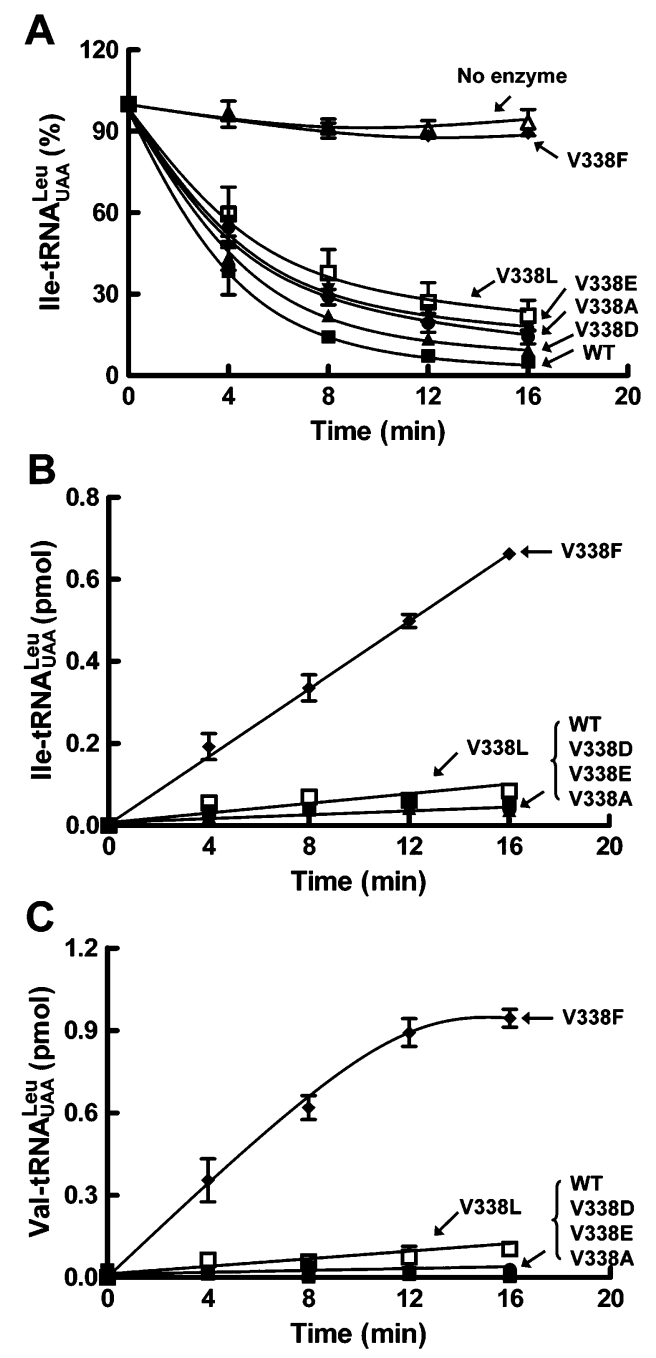

FIGURE 4: Wild type and V338 mutant LeuRS editing and mischarging activity. (A) Editing of Ile-tRNA ${ }^{\text {Leu }}$. Each editing reaction was carried out in the presence of about $0.2 \mu \mathrm{M}$ in vitro transcribed $\left[{ }^{3} \mathrm{H}\right]-$ Ile-tRNA ${ }^{\mathrm{Leu}}$ and $10 \mathrm{nM}$ enzyme. (B) Mischarging of isoleucine to in vitro transcribed tRNA ${ }^{\mathrm{Leu}}$. Each reaction was carried out in the presence of $4 \mu \mathrm{M}$ in vitro transcribed tRNA ${ }^{\mathrm{Leu}}$, $20 \mu \mathrm{M}$ isoleucine $(150 \mu \mathrm{Ci} / \mathrm{mL})$, and $0.7 \mu \mathrm{M}$ enzyme. (C) Mischarging of valine to tRNA ${ }^{\text {Leu. }}$. Each reaction was carried out in the presence of $4 \mu \mathrm{M}$ in vitro transcribed tRNA ${ }^{\text {Leu }}, 20 \mu \mathrm{M}$ valine $(150 \mu \mathrm{Ci} / \mathrm{mL})$, and $5 \mu \mathrm{M}$ enzyme. Symbols are represented as

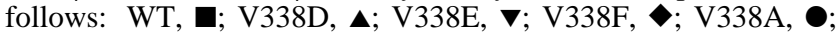
and V338L, $\square$.

position and valine at the 338 position are biologically optimal.

Adaptation of Amino Acid Specificity in Coevolved Editing Sites. The conserved $\mathrm{Arg}^{249}$ is located within the threoninerich region $\left(\mathrm{T}^{247} \mathrm{TRP}(\mathrm{D} / \mathrm{E}) \mathrm{T}\right)$ of the editing site of E. coli LeuRS. Comparison to the $E$. coli IleRS threonine-rich region $\left(\mathrm{T}^{241} \mathrm{TTPWT}\right)$ shows that this site has diverged in an idiosyncratic way and is conserved as $\mathrm{Thr}^{243}$ (Figure 1D). A neighboring site also exhibits enzyme-specific divergence where an acidic residue in LeuRS is conserved as a tryptophan in IleRS. In the T. thermophilus crystal structure, the $\mathrm{Asp}^{251}$ and $\mathrm{Arg}^{249}$ are located within $6.3 \AA$ and $3.5 \AA$ respectively of the bound amino acid side chain (Figure 5). We hypothesized that this divergence might be important to conferring substrate specificity within the editing sites of LeuRS and IleRS. 
Table 2: Apparent Kinetic Constants of Wild Type and Mutant Enzymes for Post-Transfer Editing Activity of Ile-tRNA ${ }^{\text {Leu } a}$

\begin{tabular}{lllllll}
\hline \multicolumn{1}{c}{ constants } & \multicolumn{1}{c}{ WT } & V338D & \multicolumn{1}{c}{ V338E } & V338L & V338A & V338F \\
\hline$K_{\mathrm{M}}(\mu \mathrm{M})$ & $0.13 \pm 0.06$ & $0.2 \pm 0.08$ & $0.2 \pm 0.03$ & $0.09 \pm 0.01$ & $0.2 \pm 0.02$ & $0.09 \pm 0.001$ \\
$k_{\text {cat }}\left(\mathrm{s}^{-1}\right)$ & $0.4 \pm 0.07$ & $0.3 \pm 0.06$ & $0.2 \pm 0.01$ & $0.09 \pm 0.003$ & $0.5 \pm 0.02$ & $1.19 \times 10^{-4} \pm 0.04 \times 10^{-4}$ \\
$k_{\text {cat }} / K_{\mathrm{M}}\left(\mu \mathrm{M}^{-1} \mathrm{~s}^{-1}\right)$ & 3.0 & 1.4 & 1.0 & 1.0 & 2.5 & 0.001 \\
\hline
\end{tabular}

${ }^{a}$ These measured kinetic parameters are apparent values.
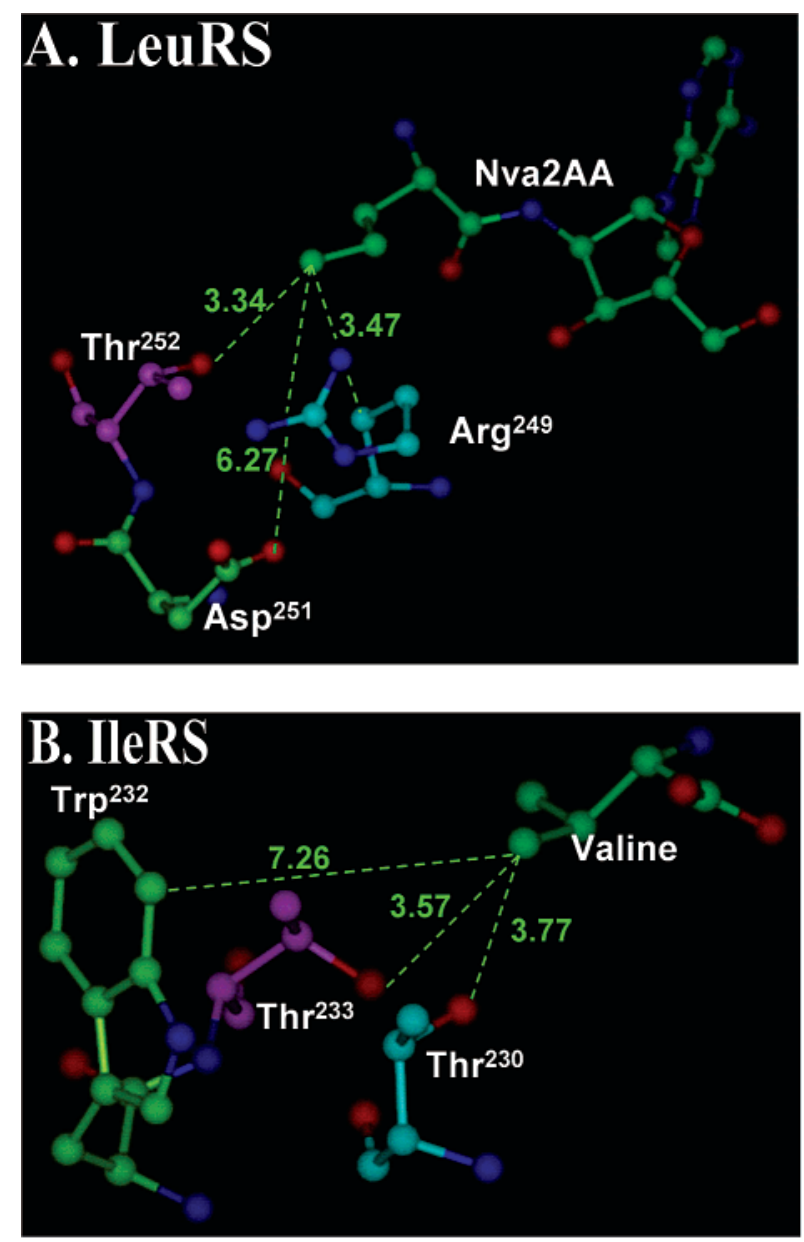

FIGURE 5: Structural analysis of the threonine-rich region within the LeuRS and IleRS CP1 domain editing site. (A) Editing site of T. thermophilus LeuRS. The carbon atoms are colored as follows: $\mathrm{Arg}^{249}$, light blue; $\mathrm{Asp}^{251}$, green; $\mathrm{Thr}^{252}$, pink; and Nva2AA, green. Oxygen and nitrogen atoms are colored in red and blue respectively. The shortest distance between each residue and the norvaline ligand is marked in a dashed green line measured in angstroms. (B) Editing site of T. thermophilus IleRS. The carbon atoms are colored as follows: valine substrate, green; $\mathrm{Thr}^{230}$, light blue; $\operatorname{Trp}^{232}$, yellow green; and $\mathrm{Thr}^{233}$, pink. Oxygen and nitrogen atoms are colored in red and blue respectively. The shortest distance $(\AA)$ between each residue and the valine ligand is marked by a dashed green line. This model was based on the X-ray crystal structure published by Nureki et al. (8).

In E. coli LeuRS, $\operatorname{Arg}^{249}$ corresponds to a threonine in IleRS and $\mathrm{Asp}^{251}$ is a tryptophan in IleRS. We swapped $\mathrm{Arg}^{249}$ and $\mathrm{Asp}^{251}$ in LeuRS with threonine (R249T) and tryptophan $(\mathrm{D} 251 \mathrm{~W})$ respectively. We also created a double mutant (R249T/D251W) that incorporated both mutations into LeuRS. Mischarging activity for isoleucine was tested to identify mutation-dependent editing defects. The R249T mutant LeuRS (Figure 6A) and the double mutant (data not shown) displayed higher mischarging activities for isoleucine, while the D251W mutant LeuRS retained its fidelity similar
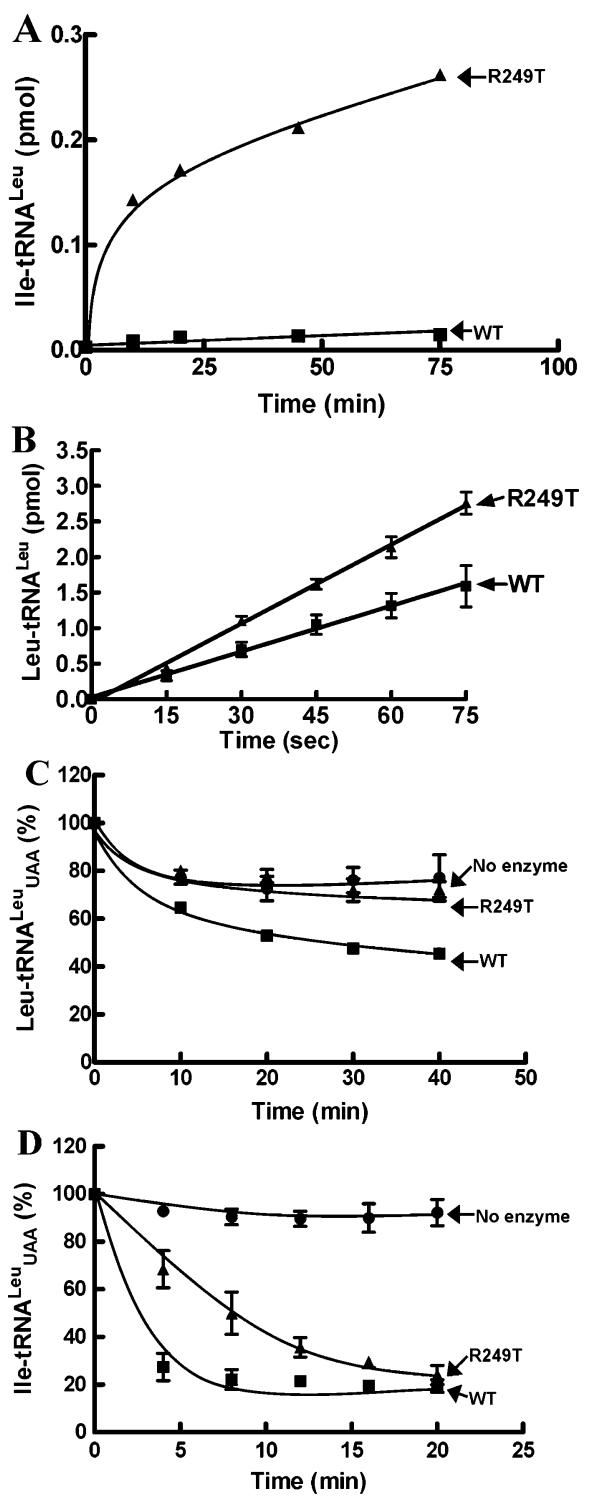

FIGURE 6: Aminoacylation and editing activities of wild type and the R249T mutant LeuRS. (A) Isoleucine misaminoacylation. Reactions are carried out in the presence of $1.7 \mu \mathrm{M}\left[{ }^{3} \mathrm{H}\right]$-isoleucine $(150 \mu \mathrm{Ci} / \mathrm{mL}), 4 \mathrm{mg} / \mathrm{mL}$ crude $\mathrm{tRNA}^{\mathrm{Leu}}$, and $5 \mu \mathrm{M}$ enzyme at room temperature. (B) Initial leucylation velocities. Reactions are carried out in the presence of $21.8 \mu \mathrm{M}\left[{ }^{3} \mathrm{H}\right]$-leucine $(166 \mu \mathrm{Ci} / \mathrm{mL}), 2 \mathrm{mg} /$ $\mathrm{mL}$ crude $E$. coli $\mathrm{tRNA}^{\mathrm{Leu}}$, and $10 \mathrm{nM}$ enzyme at $30{ }^{\circ} \mathrm{C}$. (C) Hydrolytic editing of in vitro transcribed Leu-tRNA ${ }^{\text {Leu. }}$. Reactions are carried out in the presence of $0.8 \mu \mathrm{M}$ in vitro transcribed $\left[{ }^{3} \mathrm{H}\right]-$ Leu-tRNA ${ }^{\text {Leu }}$ and $10 \mathrm{nM}$ enzyme at room temperature. (D) Hydrolytic editing of in vitro transcribed Ile-tRNA ${ }^{\text {Leu }}$. Reactions are carried out in the presence of $0.8 \mu \mathrm{M}$ in vitro transcribed $\left[{ }^{3} \mathrm{H}\right]-$ Ile-tRNA ${ }^{\text {Leu }}$ and $10 \mathrm{nM}$ enzyme at room temperature. The enzymes are represented by symbols as follows: wild type, $\mathbf{\square}$; R249T, $\mathbf{\Delta}$; no enzyme, $\bullet$. The error bars are based on reactions that were repeated at least in triplicate and are present for each point, although nominal in some cases.

to wild type LeuRS (data not shown). The leucylation activities were similar for each of the mutants (See Sup- 
porting Information.) The initial velocities for the mischarging R249T mutant LeuRS are shown in Figure 6B. These results suggest that the substituted threonine in LeuRS blocks isoleucine from the editing site. It is possible that this site in IleRS plays a role in blocking cognate isoleucine from being hydrolyzed from correctly charged Ile-tRNA ${ }^{\text {Ile }}$, similar to the role of $\mathrm{Thr}^{252}$ in LeuRS editing specificity.

Charged and mischarged in vitro transcribed tRNA ${ }^{\text {Leu, }}$, which was aminoacylated respectively with cognate leucine and noncognate isoleucine, was isolated and used to test the wild type and mutant LeuRSs' post-transfer editing activities. Over a long incubation period, none of the mutants hydrolyzed correctly charged Leu-tRNA ${ }^{\mathrm{Leu}}$ at levels greater than the wild type LeuRS (Figure 6C and data not shown). As would be expected, the low-fidelity R249T single mutant (Figure 6D) and R249T/D251W double mutant LeuRSs showed decreased hydrolysis of mischarged Ile-tRNA ${ }^{\text {Leu }}$ compared to the wild type enzyme. This weakened editing activity facilitates stable formation of mischarged IletRNA ${ }^{\text {Leu }}$.

\section{DISCUSSION}

The homologous CP1 domains of LeuRS, IleRS, and ValRS are proposed to have arisen from the same common ancestor (25). As protein synthesis became more complex and required higher fidelity, evolutionary pressures added hydrolytic editing activities and successively crafted unique specificities for LeuRS, IleRS, and ValRS. Thus, remnants of the ancestral active site were likely retained while idiosyncratic features were introduced to confer or enhance specificity. Since these three aaRSs share similar editing mechanisms, residues that are involved in catalysis or bind the common part of the editing substrate would be expected to be conserved across LeuRS, IleRS, and ValRS. This includes, for example, a universally conserved aspartic acid that anchors the common amino moiety of the amino acid of the bound editing substrate $(13,14)$. In addition, a second aspartic acid that is shared by ValRS and bacterial LeuRSs interacts with the central region that includes the ribose and amino acid backbone of the bound editing substrate $(14,15)$. However, because of differences in the side chains of leucine, isoleucine, and valine as well as the idiosyncratic challenges produced by different sets of targeted noncognate amino acids, the respective amino acid binding pockets would require divergence to provide appropriate substrate specificities within each of the LeuRS, IleRS, and ValRS editing sites.

Our mutational work shows that swapping the conserved arginine residue from LeuRS to a conserved threonine that is found at the corresponding position of IleRS altered specificity. The R249T LeuRS mutant's decrease in editing activity of Ile-tRNA ${ }^{\mathrm{Leu}}$ suggests that the binding of isoleucine might be partially blocked. The X-ray crystal structure of LeuRS bound to the unbranched norvalyl-based editing substrate analogues (14) shows that the $\beta$-methylene group of $\mathrm{Arg}^{249}$ is $3.47 \AA$ (Figure 5A) from the $\gamma$-methyl group of norvaline in the amino acid binding pocket of the editing site. This $\beta$-position of arginine in LeuRS would correspond with the branched site of the conserved threonine side chain in IleRS. The cocrystal structure of T. thermophilus IleRS CP1 domain with a valine ligand (26) also shows that the distance between the hydroxyl group of $\mathrm{Thr}^{230}$ (equivalent to $\mathrm{Thr}^{243}$ of E. coli IleRS) and one of the branched methyl groups of the valine's side chain is $3.57 \AA$ (Figure 5B). Thus, we hypothesized that the conserved threonine in the IleRS editing site helps block isoleucine to prevent hydrolysis of the correctly charged Ile-tRNA ${ }^{\text {Ile }}$ during the editing reaction, which primarily targets misactivated and mischarged valine. Indeed, it has been published recently that the T. thermophilus IleRS $\mathrm{Thr}^{230}$ blocks isoleucine from binding to the editing site (27). An alanine substitution at this site leads to rapid hydrolysis of the cognate Ile-tRNA ${ }^{\mathrm{Il}}$. This suggests that the LeuRS and IleRS editing sites have diverged at this particular residue to confer specificity of their respective substrates.

Interestingly, a reverse mutation in E. coli IleRS at this site (T243R), which substitutes an arginine found at that site in LeuRS for the conserved threonine, also disrupts editing to a level that causes growth defects (28). In vitro analysis showed that valine was mischarged to tRNA ${ }^{\text {Ile }}$ due to the decrease in the dominant pre-transfer editing mechanism. Since the pre- and post-transfer editing activities appear to share an amino acid binding pocket in the editing site, it is likely that the arginine side chain blocks amino acid binding of the misactivated adenylate in the editing site pocket to decrease fidelity.

Mutation of the second idiosyncratic residue, LeuRS $\mathrm{Asp}^{251}$, failed to significantly alter the enzyme's editing activities. The X-ray cocrystal structure of $T$. thermophilus LeuRS with a post-transfer editing substrate analogue (14) shows that the side chain oxygens of $\mathrm{Asp}^{251}$ are distant from the $\gamma$-methyl group of norvaline $(6.27 \AA$ and $7.14 \AA$ respectively) and thus would likely not interact directly with the amino acid side chain (Figure 5). Likewise, the corresponding $\operatorname{Tr}^{232}$ of $T$. thermophilus IleRS (equivalent to $\operatorname{Trp}^{245}$ of $E$. coli IleRS) is $7.26 \AA$ from the side chain of the bound valine (26). Interestingly, a W245E mutant in E. coli IleRS abolished editing activity (29). This is consistent with an earlier structure (8), in which the conserved $\operatorname{Trp}^{232}$ and $\mathrm{Tyr}^{386}$ formed an editing pocket that was proposed to be sufficient for valine binding, but too small for isoleucine. Changing the tryptophan to a charged residue was proposed to disrupt the hydrophobic pocket resulting in a loss of editing.

A nearly completely conserved threonine ( $\mathrm{Th}^{252}$ in E. coli LeuRS) is important to specificity in LeuRS $(12,16,17)$. The side chain clashes with the $\gamma$-methyl branch of the leucine side chain to block it from binding (14). Mutation to an alanine uncouples specificity and facilitates hydrolysis of the correctly charged Leu-tRNA ${ }^{\text {Leu }}$. The corresponding mutant in IleRS, where the completely conserved threonine is changed to alanine, did not show any alteration in editing activity (29). This would be expected because the isoleucine and valine side chains lack a $\gamma$-methyl branch. Thus, the conserved threonine residue in IleRS might represent a remnant of the divergence of IleRS and LeuRS. It also may have functionally diverged to play a more specific structural role in IleRS.

\section{ACKNOWLEDGMENT}

We thank Dr. Wei Fu for providing computational expertise. We are also grateful to Dr. R. S. Mursinna for his contribution to understanding the M336A LeuRS mutation. 


\section{SUPPORTING INFORMATION AVAILABLE}

Figures depicting leucylation activity and leucine aminoacylation of tRNA ${ }^{\text {Leu }}$ by wild type and mutant LeuRSs. This material is available free of charge via the Internet at http://pubs.acs.org.

\section{REFERENCES}

1. Martinis, S. A., Plateau, P., Cavarelli, J., and Florentz, C. (1999) Aminoacyl-tRNA synthetases: a family of expanding functions, EMBO J. 18, 4591-4596.

2. Martinis, S. A., Plateau, P., Cavarelli, J., and Florentz, C. (1999) Aminoacyl-tRNA synthetases: a new image for a classical family, Biochimie 81, 683-700.

3. Hendrickson, T. L., and Schimmel, P. (2003) Transfer RNAdependent amino acid discrimination by aminoacyl-tRNA synthetases, in Translation Mechanisms (LaPointe, J., and BrakierGingras, L., Eds.) pp 34-64, Klumer Academic/Plenum Publishers, New York.

4. Fersht, A. R. (1977) Editing mechanisms in protein synthesis. Rejection of valine by the isoleucyl-tRNA synthetase, Biochemistry 16, 1025-1030.

5. Fersht, A. R. (1998) Sieves in sequence, Science 280, 541.

6. Fersht, A. R., and Dingwall, C. (1979) Evidence for the doublesieve editing mechanism in protein synthesis. Steric exclusion of isoleucine by valyl-tRNA synthetases, Biochemistry 18, 26272631.

7. Cusack, S., Yaremchuk, A., and Tukalo, M. (2000) The 2 A crystal structure of leucyl-tRNA synthetase and its complex with a leucyladenylate analogue, EMBO J. 19, 2351-2361.

8. Nureki, O., Vassylyev, D. G., Tateno, M., Shimada, A., Nakama, T., Fukai, S., Konno, M., Hendrickson, T. L., Schimmel, P., and Yokoyama, S. (1998) Enzyme structure with two catalytic sites for double-sieve selection of substrate, Science 280, 578-582.

9. Fukai, S., Nureki, O., Sekine, S., Shimada, A., Vassylyev, D. G., and Yokoyama, S. (2003) Mechanism of molecular interactions for tRNA ${ }^{\text {Val }}$ recognition by valyl-tRNA synthetase, RNA 9, 100111.

10. Starzyk, R. M., Webster, T. A., and Schimmel, P. (1987) Evidence for dispensable sequences inserted into a nucleotide fold, Science $237,1614-1618$.

11. Lin, L., Hale, S. P., and Schimmel, P. (1996) Aminoacylation error correction, Nature 384, 33-34.

12. Mursinna, R. S., Lincecum, T. L., Jr., and Martinis, S. A. (2001) A conserved threonine within Escherichia coli leucyl-tRNA synthetase prevents hydrolytic editing of leucyl-tRNA ${ }^{\text {Leu }}$, Biochemistry 40, 5376-5381.

13. Bishop, A. C., Nomanbhoy, T. K., and Schimmel, P. (2002) Blocking site-to-site translocation of a misactivated amino acid by mutation of a class I tRNA synthetase, Proc. Natl. Acad. Sci. U.S.A. 99, 585-590.

14. Lincecum, T. L., Jr., Tukalo, M., Yaremchuk, A., Mursinna, R. S., Williams, A. M., Sproat, B. S., Van Den Eynde, W., Link, A., Van Calenbergh, S., Grøtli, M., Martinis, S. A., and Cusack, S.
(2003) Structural and mechanistic basis of pre- and posttransfer editing by leucyl-tRNA synthetase, Mol. Cell 11, 951-963.

15. Fukai, S., Nureki, O., Sekine, S., Shimada, A., Tao, J., Vassylyev, D. G., and Yokoyama, S. (2000) Structural basis for double-sieve discrimination of $\mathrm{L}$-valine from L-isoleucine and L-threonine by the complex of tRNA(Val) and valyl-tRNA synthetase, Cell 103, 793-803.

16. Mursinna, R. S., Lee, K. W., Briggs, J. M., and Martinis, S. A. (2004) Molecular dissection of a critical specificity determinant within the amino acid editing domain of leucyl-tRNA synthetase, Biochemistry 43, 155-165.

17. Mursinna, R. S., and Martinis, S. A. (2002) Rational design to block amino acid editing of a tRNA synthetase, J. Am. Chem. Soc. 124, 7286-7287.

18. Tang, Y., and Tirrell, D. A. (2002) Attenuation of the editing activity of the Escherichia coli leucyl-tRNA synthetase allows incorporation of novel amino acids into proteins in vivo, Biochemistry 41, 10635-10645.

19. Tocchini-Valentini, G., Saks, M. E., and Abelson, J. (2000) tRNA leucine identity and recognition sets, J. Mol. Biol. 298, 779-793.

20. Cazenave, C., and Uhlenbeck, O. C. (1994) RNA template-directed RNA synthesis by T7 RNA polymerase, Proc. Natl. Acad. Sci. U.S.A. 91, 6972-6976.

21. Zhai, Y., and Martinis, S. A. (2005) Two conserved threonines collaborate in the Escherichia coli leucyl-tRNA synthetase amino acid editing mechanism, Biochemistry 44, 15437-15443.

22. Martinis, S. A., and Fox, G. E. (1997) Non-standard amino acid recognition by Escherichia coli leucyl-tRNA synthetase, Nucleic Acids Symp. Ser. 36, 125-128.

23. Tukalo, M., Yaremchuk, A., Fukunaga, R., Yokoyama, S., and Cusack, S. (2005) The crystal structure of leucyl-tRNA synthetase complexed with tRNA ${ }^{\text {Leu }}$ in the post-transfer-editing conformation, Nat. Struct. Mol. Biol. 12, 923-930.

24. Fukunaga, R., and Yokoyama, S. (2005) Aminoacylation complex structures of leucyl-tRNA synthetase and tRNA ${ }^{\text {Leu }}$ reveal two modes of discriminator-base recognition, Nat. Struct. Mol. Biol. 12, 915-922.

25. Schimmel, P., and Ribas de Pouplana, L. (2001) Formation of two classes of tRNA synthetases in relation to editing functions and genetic code, Cold Spring Harb Symp. Quant. Biol. 66, 161166.

26. Fukunaga, R., Fukai, S., Ishitani, R., Nureki, O., and Yokoyama, S. (2004) Crystal structures of the CP1 domain from Thermus thermophilus isoleucyl-tRNA synthetase and its complex with L-valine, J. Biol. Chem. 279, 8396-8402.

27. Fukunaga, R., and Yokoyama, S. (2006) Structural basis for substrate recognition by the editing domain of isoleucyl-tRNA synthetase, J. Mol. Biol. 359, 901-912.

28. Hendrickson, T. L., Nomanbhoy, T. K., de Crecy-Lagard, V., Fukai, S., Nureki, O., Yokoyama, S., and Schimmel, P. (2002) Mutational separation of two pathways for editing by a class I tRNA synthetase, Mol. Cell 9, 353-362.

29. Schmidt, E. (1996) Amino acid recognition by a class I tRNA synthetase, Ph.D. Thesis, Massachusetts Institute of Technology, Cambridge, Massachusetts.

BI061778L 